Eeva M. Ryhänen ${ }^{1}$, Helena Leijon², Saara Metso3 ${ }^{3}$, Eija Eloranta ${ }^{4}$, Pirkko Korsoff ${ }^{5}$, Petteri Ahtiainen ${ }^{6}$, Päivi Kekäläinen ${ }^{7}$, Marjo Tamminen $^{8}$, Raija Ristamäki ${ }^{9}$, Otto Knutar ${ }^{10}$, Eliisa Löyttyniemi ${ }^{11}$, Leo Niskanen ${ }^{1}$, Ilkka Heiskanen ${ }^{12}$, Mika Väisänen ${ }^{12}$, Matti J. Välimäki ${ }^{1}$, Caj Haglund ${ }^{13}$, Johanna Arola $^{2}$, Camilla Schalin-Jäntti ${ }^{1}$

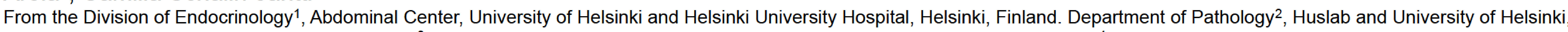

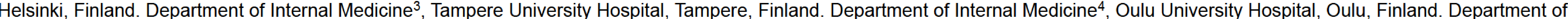

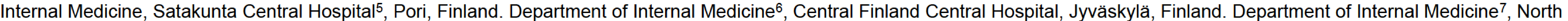
Carelia Central Hospital, Joensuu, Finland. Department of Internal Medicine ${ }^{8}$, Kymenlaakso Central Hospital, Kotka, Finland. Department of Oncology ${ }^{9}$, Turku University Hospital, Turku, Finland. Department of Internal Medicine, Vaasa Central Hospital ${ }^{10}$, Vaasa, Finland. Department of Biostatistics ${ }^{11}$, University of Turku, Turku, Finland. Department of Surgery ${ }^{12}$, Helsinki University Hospital, Helsinki, Finland, and Research Programs Unit ${ }^{13}$, Translational Cancer Biology, University of Helsinki, Helsinki, Finland

\section{INTRODUCTION AND OBJECTIVES}

Parathyroid carcinoma $(\mathrm{PC})$ is a very rare endocrine cáncer, and a rare cause of primary hyperparathyroidism (PHPT). Data on PC is still scarce. The diagnosis of $\mathrm{PC}$ is challenging and the incidence seems to increase. Our aims were:

1)to investigate whether the incidence of PC has increased in Finland

2) to better characterize PC by comparing it to atypical parathyroid adenoma (AA) and parathyroid adenoma (A)

3) to identify potential early markers for a) malignancy b) recurrent $\mathrm{PC}$, by assessing clinical, histological, immunochemical, surgical and other treatment characteristics as well as prognosis of $\mathrm{PC}, \mathrm{AA}$ and $\mathrm{A}$

\section{PATIENTS AND METHODS}

Study group We re-examined all parathyroid carcinomas (PC; $\mathrm{n}=32$ ) diagnosed in Finland during years 2000-2011. This group was compared to 28 patients with atypical parathyroid adenoma (AA) and 72 age- and gender matched patients with parathyroid adenoma (A) from Helsinki University Hospital.

Histological re-evaluation The diagnose of PC was re-examined in all primary tumors according to the WHO 2004 criteria (J.A. and E.R.) . Of the original $39 \mathrm{PC}$ cases, three were re-classified as thyroid carcinoma and excluded. Four other cases lacked tumor invasion and were re-classified as AA.

Data collection Clinical data, laboratory measurements as well as data on surgical and other treatments, and recurrences were collected retrospectively from medical records and revised by E.R. Survival data and causes of death of the study group, as well as population data were obtained from Statistics Finland.

Mean age of the 32 PC patients (44\% men) was 61 (17-83) yrs. The mean follow-up time was 6.7 (range 2.0-13.9) yrs.

Immunohistochemical staining for Ki-67, parafibromin and PTH were performed using TMA (Tissue Micro Array) analysis. For TMA, 3-6 cores were sampled from representative areas of primary tumor blocks and inserted into a recipient paraffin block.TMA blocks were cut into $4 \mu \mathrm{m}$ slices and processed for Ki-67, parafibromin and PTH stainings. Ki-67 was assessed by Immunoratio (Tuominen et al.). PTH- and parafibromin stains were assessed by two independent investigators (H.L. and E.R.)

Ethics The study protocol was approved by the institutional review board of Helsinki University Hospital, by the ethics committees of the four other Finnish University Hospitals, by National Supervisory Authority for Welfare and Health in Finland,and by the National Institute for Health and Welfare in Finland.

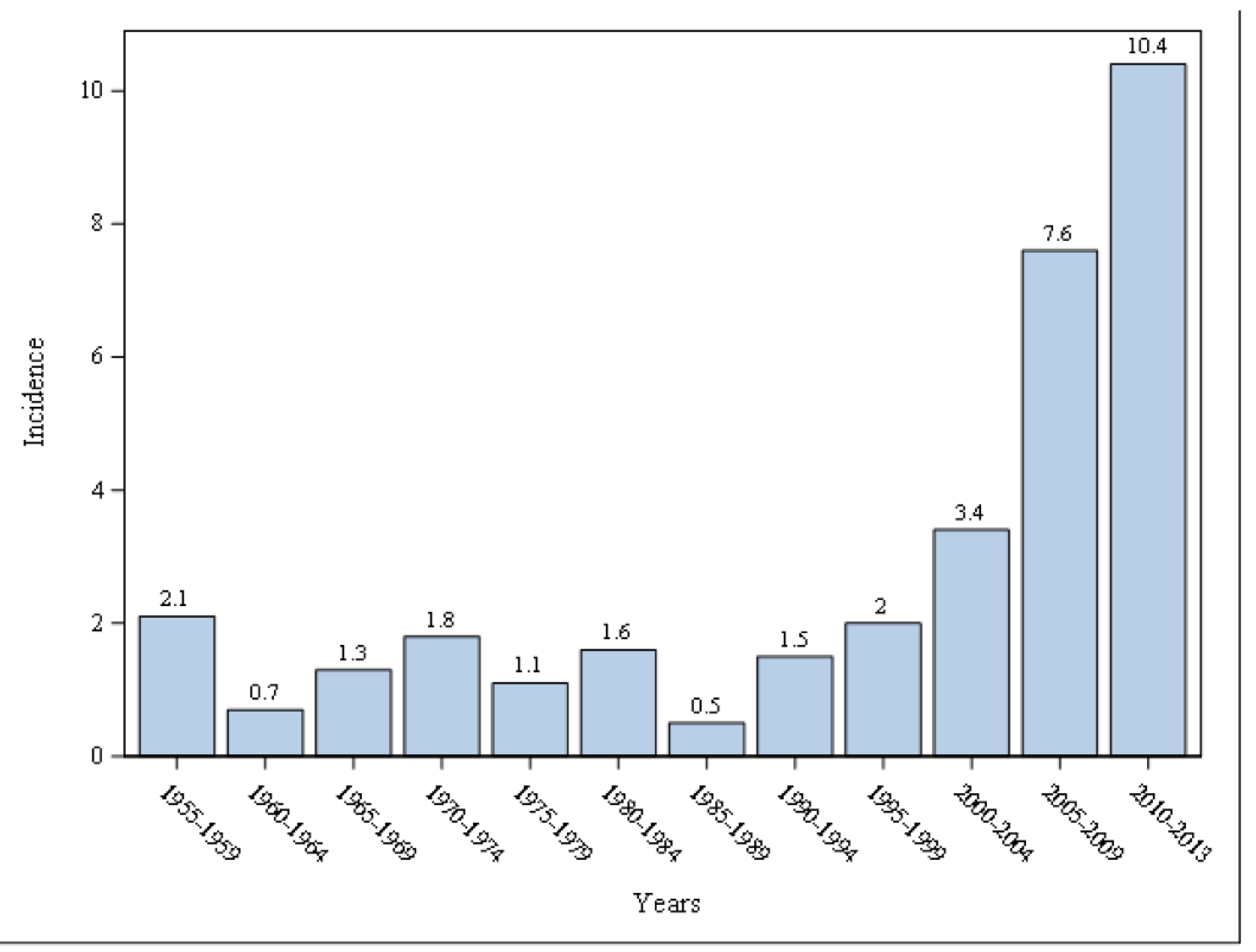

FIGURE Incidence of PC in Finland between 1955 - 2013 per 10,000,000 inhabitants per year.

\section{RESULTS}

PC patients had higher serum ionized calcium (Ca-ion) and PTH levels compared to AA and A groups: $1.76(1.61-1.97) \mathrm{mmol} / 1 \mathrm{vs.} 1.56(1.50-1.70)$ vs. $1.44(1.38-1.51)$, (median and IQR, reference range 1.15-1.30), and 989 (461-1518) vs. $355 \quad(149$ 959) vs $160(122-251) \mathrm{ng} / 1$, reference range $15-65 \mathrm{ng} / 1), P<0.001$ for both. $\mathrm{PC}$ and AA demonstrated increased tumor size compared to A $(P<0.001)$

PC patients had more often both bone and renal manifestations than AA and A patients $(28 \%$ vs $7 \%$ vs $6 \%$, respectively, $P=0.004)$ and were more often hospitalized for severe hypercalcemia $(44 \%$ vs $22 \%$ vs $3 \%, P<0.001)$. Twelve $(37.5 \%)$ PC patients also had a benign parathyroid tumor in their medical history or during follow-up.

Histopathological characteristics of $\mathrm{PC}$ were chief cells $(P=0.004)$, diffuse growth pattern $(P<0.001)$, increased mitotic activity $(P<0.001)$, nuclear atypia $(P=0.003)$, higher Ki-67 $(P<0.001)$, and weaker/negative parafibromin stain $(P<0.001)$. In the PC group, $9.4 \%$ had residual disease, and $21 \%$ of those cured had a recurrence. Five had metastatic disease. Although $\mathbf{1 2 . 5 \%}$ died of PC during follow-up, overall mortality did not differ between the subgroups $(P=0.94)$.

Nearly half (47\%) of PC patients had two or more operations. External neck radiation and chemotherapy was given to $22 \%$ and $13 \%$ of PC patients, respectively. At diagnosis, lymph node status $(P<0.03)$, tumor necrosis $(P=0.006)$, mitotic activity $(P=0.045)$, and parafibromin stain $(P=0.01)$ differed between recurrent and non-recurrent PCs. All recurrent tumors presented with vascular invasion $(P=0.29)$

\section{CONCLUSIONS}

We present a nationwide cohort of PC from 2000-2011 ( $\mathrm{n}=32)$, and demonstrate a five-fold increase in the incidence of PC in Finland in line with studies from USA and Australia (Lee et al, Brown et al).

PC associates with clinically more severe PHPT compared to AA or A, and has distinct histopathological features. Nearly $40 \%$ of PC patients have additional benign parathyroid tumors, and $47 \%$ of PC patients undergo 2 or more neck surgeries, increasing the risk for surgical complications.

PC should be suspected in all PHPT patients having S-Ca-ion $>1.70 \mathrm{mmol}$, as none of the benign cases reached this threshold. However, PC may also be characterized by milder hypercalcemia.

Histopathologic features of aggressive, recurrent PC include lymph node metastasis, vascular invasion, necrosis, negative parafibromin staining and $\mathrm{Ki} 67>5 \%$.

In PC, recurrence rate ( $21 \%$ in $6.7 \mathrm{yrs}$ follow-up) is lower than that other previously reported (Harari et al, Talat et al), and overall survival was not different from that of patients with adenomas.

\section{References}

Lee PK, Jarosek SL, Virnig BA, Evasovich M \& Tuttle TM 2007 Trends the incidence and treatment of parathyroid cancer in the United States. Cancer 109 1736-1741.

Brown S, O'Neill C, Suliburk J, Sidhu S, Sywak M, Gill A, Robinson B \& Delbridge L 2011 Parathyroid carcinoma: increasing incidence and changing presentation. ANZ Journal of Surgery 81 528-532. Harari A, Waring A, Fernandez-Ranvier G, Hwang J, Suh I, Mitmaker E, Shen W, Gosnell J, Duh QY \& Clark O 2011 Parathyroid carcinoma: a 43year outcome and survival analysis. The Journal of clinical endocrinolog and metabolism 96 3679-3686.

Sadler C, Gow KW, Beierle EA, Doski JJ, Langer M, Nuchtern JG, Vasudevan SA \& Goldfarb M 2014 Parathyroid carcinoma in more than 1,000 patients: A population-level analysis. Surgery 156 1622-9; discussion $1629-30$

Talat N \& Schulte KM 2010 Clinical presentation, staging and long-term evolution of parathyroid cancer Annals of surgical oncology 17 2156-2174. Tuominen VJ, Ruotoistenmaki S, Viitanen A, Jumppanen M \& Isola J 2010 ImmunoRatio: a publicly available web application for quantitalive J 2010

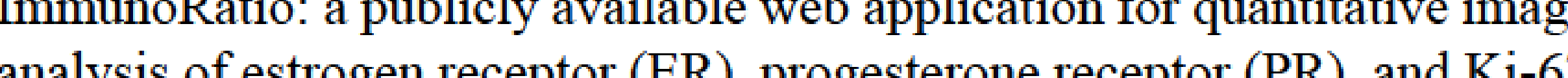
Breast cancer research : $B C R \mathbf{1 2} \mathrm{R} 56$. 